\title{
Research and Application of FTA and Petri Nets in Fault Diagnosis in the Pantograph-Type Current Collector on CRH EMU Trains
}

\author{
Long-long Song, ${ }^{1}$ Tai-yong Wang, ${ }^{1,2}$ Xiao-wen Song, ${ }^{3}$ Lei $\mathrm{Xu}^{3}{ }^{3}$ and De-gang Song ${ }^{3}$ \\ ${ }^{1}$ School of Mechanical, Electronic and Control Engineering, Beijing Jiaotong University, Beijing 100044, China \\ ${ }^{2}$ School of Mechanical Engineering, Tianjin University, Tianjin 300192, China \\ ${ }^{3}$ R\&D Center, CSR Qingdao Sifang Co., Ltd., Qingdao 266111, China
}

Correspondence should be addressed to Long-long Song; song_bjtu@163.com

Received 28 May 2015; Revised 8 September 2015; Accepted 14 September 2015

Academic Editor: Yuanchang Xie

Copyright (C) 2015 Long-long Song et al. This is an open access article distributed under the Creative Commons Attribution License, which permits unrestricted use, distribution, and reproduction in any medium, provided the original work is properly cited.

A fault tree is established based on structural analysis, working principle analysis, and failure mode and effects analysis (FMEA) of the pantograph-type current collector on the Chinese Rail High-Speed Electric Multiple Unit (CRH EMU) train. To avoid the deficiencies of fault tree analysis (FTA), Petri nets modelling is used to address the problem of data explosion and carry out dynamic diagnosis. Relational matrix analysis is used to solve the minimal cut set equation of the fault tree. Based on the established state equation of the Petri nets, initial tokens and enable-transfer algorithms are used to express the fault transfer process mathematically and improve the efficiency of fault diagnosis inferences. Finally, using a practical fault diagnosis example for the pantographs on CRH EMU trains, the proposed method is proved to be reasonable and effective.

\section{Introduction}

In recent years, high-speed electric multiple unit (EMU) trains have become an important mode of transportation in China. The Chinese Rail High-Speed (CRH) EMU train is a large-scale intelligent system with complex structures. The reliability of an EMU train is directly related to the safety and efficiency of the high-speed, heavy-load rail transportation system [1]. The high-voltage traction drive system is one of the most critical aspects of an EMU train system. As a critical subsystem of the high voltage traction system, the pantograph-type current collector plays an important role in providing power for an EMU train by coupling directly with the electric catenary wire $[2,3]$. Efficient and accurate fault diagnosis for the pantograph is vital for the safe and stable operation of CRH EMU trains.

Complexity is defined as a state in which many different parts (hardware, software, organizational, and human elements) are related to each other in an interconnected manner [4]. It is defined here in two forms: structural complexity and fault complexity. The CRH EMU train system is complex both in its structure and in its failure modes. Though the structure of the pantograph seems relatively simple, the working principles and failure modes of pantographs are surprisingly complex. For example, there are many complex potential failure modes for a pantograph, which are related to mechanical structures, electrical facilities, pneumatic transmission, network control, and many other aspects. The operation of the pantograph on a CRH EMU train is automatically controlled by microprocessors. The pantograph system involves mechanical structures such as guide bars, electrical equipment such as the traction converter, and rubber products such as the base insulator. The lifting process is controlled by air pressure. The complex failure modes of the pantograph can involve cracks, breaks, fatigue, pitting, wear, and discharge breakdown. The relationship between different failures is complex and uncertain. Consequently, the pantograph system of a CRH EMU train is treated as a complex system. A useful approach combining fault tree analysis and Petri nets theory is applied to deal with the complexity of fault diagnosis in the pantograph system. 
Fault tree analysis (FTA) is a useful analytical tool for identifying and classifying hazards and calculating system reliability for both simple and complex engineering systems [5]. It is a systematic way to assess the reliability of complex systems both qualitatively and quantitatively [6]. Much research has been conducted and outcomes include extended fuzzy FTA (FFTA) methodology in the petrochemical process industry in fuzzy environments [7]; the use of a fuzzylogic-based reliability approach to evaluate basic events in fault tree analysis for nuclear power plant probabilistic safety assessment [8]; FFTA applied to reducing uncertainty in expert judgment in the safety barriers analysis of an offshore drilling system [9]; and the use of fault tree analysis and analytic hierarchy processes to analyze the risks associated with the use of shield tunnel boring machines (TBMs) [10].

However, the modelling power of FTA is limited to the static evaluation of a single criterion at a time and FTA is not capable of describing dynamic system behavior with redundant components, degraded system states, and repair or test activities [11]. Leveson and Stolzy proposed the safety analysis of dynamic systems using time Petri nets [12]. Bobbio et al. provided an algorithm to convert a parametric fault tree (PFT) into a class of high-level Petri nets to exploit the modelling power and flexibility of the stochastic well-formed nets (SWN) formalism [13]. Robidoux et al. used a framework named dynamic reliability block diagrams (DRBD) for modelling the dynamic reliability behavior of computerbased systems and presented an algorithm that automatically converted a DRBD model into a colored Petri net [14]. With increasing complexity in the relationship between system structures and faults, fault trees become more difficult to establish. Problems relating to the Nondeterministic Polynomial (NP), which can lead to combinatorial explosion as the numbers of calculations grow exponentially, appear when solving the minimal cut sets. Makajic-Nikolic et al. proposed an approach to cutting the number of minimal cut sets in a fault tree using reverse Petri nets [15]. There have also been some studies on the application of FTA and Petri nets in transportation systems. For example, Wang et al. proposed reliability modelling and evaluation issues of electric vehicle motors by using FT FTA-based extended stochastic Petri nets [16]; Nguyen et al. combined a Petri nets (PN) method with extensions of FTA to adapt to dynamic systems and applied the method to a satellite-based railway system [17]; and Song et al. applied T-S Fuzzy theory and FTA to diagnose pantograph faults in a multisource heterogeneous knowledge environment [18].

Some other mathematical/statistical methods such as fuzzy reasoning and Bayesian Network (BN) also have been successfully used in the railways risk and safety domain. For example, An et al. proposed a railway risk management system for railway risk analysis using fuzzy reasoning approach and fuzzy analytical hierarchy decision making process $[19,20]$; Noori and Jenab developed a fuzzy Bayesian traction control system for rail vehicles with speed sensors in intelligent transportation systems [21]; Muttram applied a Safety Risk Model combining fault tree analysis and cause/consequence techniques to predict residual levels of railway safety risk [22]; Bouillant et al. developed a decision support tool based on Bayesian Networks to evaluate, compare, and optimize various operating and maintenance strategies of Paris metro underground rails [23]; Xie et al. introduced the Bayesian inference and investigated the application of a Bayesian ordered probit (BOP) model in driver's injury severity analysis and verified that BOP model could produce more reasonable parameter estimations and better prediction performance than the ordered probit (OP) model [24]; Bernardi et al. generated Repairable Fault Tree and Bayesian Network models for railway modelling by a model-driven approach called DAM-Rail approach [25]; Mahboob and Straub compared fault tree and Bayesian Networks for modeling safety critical components in railway systems [26]; and Washington and Oh incorporated Bayesian methodology with expert judgment for countermeasure effectiveness under uncertainty and applied the approach in improving the safety of railroad crossings [27].

From the above, it appears that the conventional analysis approaches, such as FTA, Petri nets (PN), fuzzy reasoning, and $\mathrm{BN}$, have been widely used in the railways risk and safety analysis field. However, each approach has its own advantages and limitations. Fault tree, Petri nets, and BN have a strong similarity in many aspects. Fault tree can be established more easily only when the cause-and-effect relationships between events were clear. But it suffers severe limitations of statics structure and uncertainty handling. Fuzzy reasoning and $\mathrm{BN}$ are effective tools for quantitative analysis because they are based on probabilistic and uncertain knowledge. $\mathrm{BN}$ approach allows dealing with issues such as prediction or diagnosis, optimization, and data analysis of feedback experience [28]. It is more often used in prediction because of good quantitative analysis ability, but the training process of $\mathrm{BN}$ is complex. And the dependent probabilities among events are required in $\mathrm{BN}$ approach which may be difficult to obtain in some cases. Petri net $(\mathrm{PN})$ is also a powerful and widely used graphical and mathematical modelling tool for the description of sequence dependent behaviors of dynamic systems. When the failure status of a system evolves from one subsystem/component to other subsystems/components, Petri net modelling is more intuitive and more effective to describe the process by carrying mathematical matrix computations, which is easier to handle by computer. At the same time, it is more suitable for large complex systems because the modelling process is simplified by eliminating repeat basic events and reducing the building elements. Through the above analysis, FTA's translation into Petri nets is more suitable in this paper to analyze the dynamic behaviors of failure statuses of Pantograph-Type Current Collector on CRH EMU trains mathematically as far as the extension, fast modelling and dynamic behavior analysis of the system are mainly concerned.

Studies on reliability and failure mode analysis of $\mathrm{CRH}$ EMU trains and their subsystems have recently begun. The focus of the research has turned from design and manufacture to maintenance management. FTA and Petri nets approaches are applicable and useful analysis tools in the risk management of complex engineering systems, but there are some deficiencies in their application. Considerable research has been conducted on these problems. The main aim of 
this paper is to extend FTA and Petri nets methodology to maintenance and fault diagnosis in CHR EMU train systems. This section introduces some existing applications of FTA and Petri nets in a range of industries. Structural analysis, working principles, failure mode and effects analysis (FMEA), and FTA modelling of pantographs are outlined in Section 2. Petri nets modelling of the fault tree and the use of relational matrix analysis in solving minimal cut sets are provided in Section 3. In Section 4, the place mark and enable-transfer algorithm of the Petri nets and an actual case study on dynamic transition and diagnosis of pantograph diagnostics are provided. The last section emphasises the highlights of this research.

\section{Structural Analysis and Fault Tree Modelling of Pantograph}

2.1. Structural Analysis and Working Principle of Pantograph. The pantograph of a CRH EMU train is fitted on the roof of the train and is essential to allow the train to get power from the main overhead wires. A CRH EMU train is an eight-car multiple unit configured with four motorised cars and four trailer cars. There are two power units in all and each power unit consists of two power cars and two trailer cars, arranged in T-M-M-T mode. DSA250 pantographs are fitted on number 4 and number 6 cars. Their maximum lifting height is $3000 \mathrm{~mm}$ and the width of the head is $1990 \mathrm{~mm}$. In normal operation, there will be only one pantograph collecting current, with another in a folded state. However, when two CRH EMUs are attached together, two pantographs will work simultaneously. The working principle and structural components of pantograph systems for $\mathrm{CRH}$ EMUs are shown in Figure 1. The structural parameters of the pantograph are shown in Table 1.

The pantograph is raised to access high voltage power by allowing the carbon skateboard to contact the catenary wire and descends when its compressed air supply is exhausted. The $25 \mathrm{kv}$ single-phase power alternating current (AC) from the catenary is transferred to the traction transformer from the high-voltage electrical equipment by the pantograph, which outputs $1500 \mathrm{~V}$ single-phase AC power to the traction converter. A pulse rectifier converts the single-phase AC into direct current (DC) and outputs 2500-3000 V DC to the traction inverter through the DC circuit. Then the traction inverter outputs three-phase AC power, where the voltage and frequency are all adjustable, to drive the traction motor.

When the pantograph rises, air is compressed into the drive cylinder through the cushion valve and the cylinder piston moves left, overcoming the pressure of the reset spring. Then the lower arm rises and rotates clockwise under the action of the guide bar and the spring. At the same time, the upper arm also rises with the drive of the top guide bar.

When the pantograph descends, the compressed air is removed from the drive cylinder through the cushion valve. Then the piston is pushed to the right with the reset spring releasing pressure. The guide bar also moves right to force the lower arm to rotate anticlockwise, thus forcing the upper arm to descend.

The collector head contacts the catenary wire when the pantograph rises. Current is led to the bottom frame through the collector head, the upper arm, and the pushrod. The power cable installed on the bottom frame then leads the current to the vehicle. Since current will flow through the entire pantograph frame in the power supply state, all the pantograph hinges are equipped with bridge connections to prevent the current damaging the bearings.

The performance of the pantograph largely depends on contact pressure. If there is too little pressure, contact resistance will vary easily, resulting in poor contact and arcing. However, too much pressure will increase the friction, aggravating wear on the carbon skateboard and wires and reducing the life of the carbon skateboard.

2.2. Failure Mode and Effects Analysis (FMEA) of Pantograph System. Pantographs on CRH EMU trains are installed on the roof, exposed to the environment. The working environment is complex, volatile, and sometimes very harsh. There are many and complex ways in which the pantograph can fail, which are related to mechanical structures, electrical facilities, pneumatic transmission, network control, and many other factors. The normal operation of the pantograph on a CRH EMU train is automatically controlled by microprocessors. The pantograph system involves mechanical structures such as guide bars, electrical equipment such as the traction converter, and rubber products such as the base insulator. The lifting process is controlled by air pressure. Because pantographs connect directly with the extra high voltage (EHV) catenary wire, they can be easily damaged by partial high voltage discharges. By classifying and analyzing fault tracking records, the most common pantograph failure modes are summarized as follows.

(i) Failure Mode 1. Pantograph rises to an abnormal position.

Probable cause:

When two EMUs attach together, the space between the two pantographs is less than $190 \mathrm{~m}$.

(ii) Failure Mode 2. Pantograph rises normally, while the monitor (MON) does not show it correctly.

Probable causes:

(a) The pressure sensors are not operating correctly.

(b) The pressure switches have failed.

(iii) Failure Mode 3. Pantograph cannot rise.

Probable causes:

(a) Electricity generation system (EGS) is closed.

(b) Vacuum circuit breaker (VCB) is closed.

(c) Pressure in the auxiliary air cylinder is too low.

(d) [Pantograph.VCB] NFB is in the OFF position.

(e) [Pantograph Rising] NFB is in the OFF position.

(f) Pantograph pressurised air ducts leak.

(g) White air ducts connected to the pantograph leak.

(h) Air ducts in the pantograph control valve board leak. 


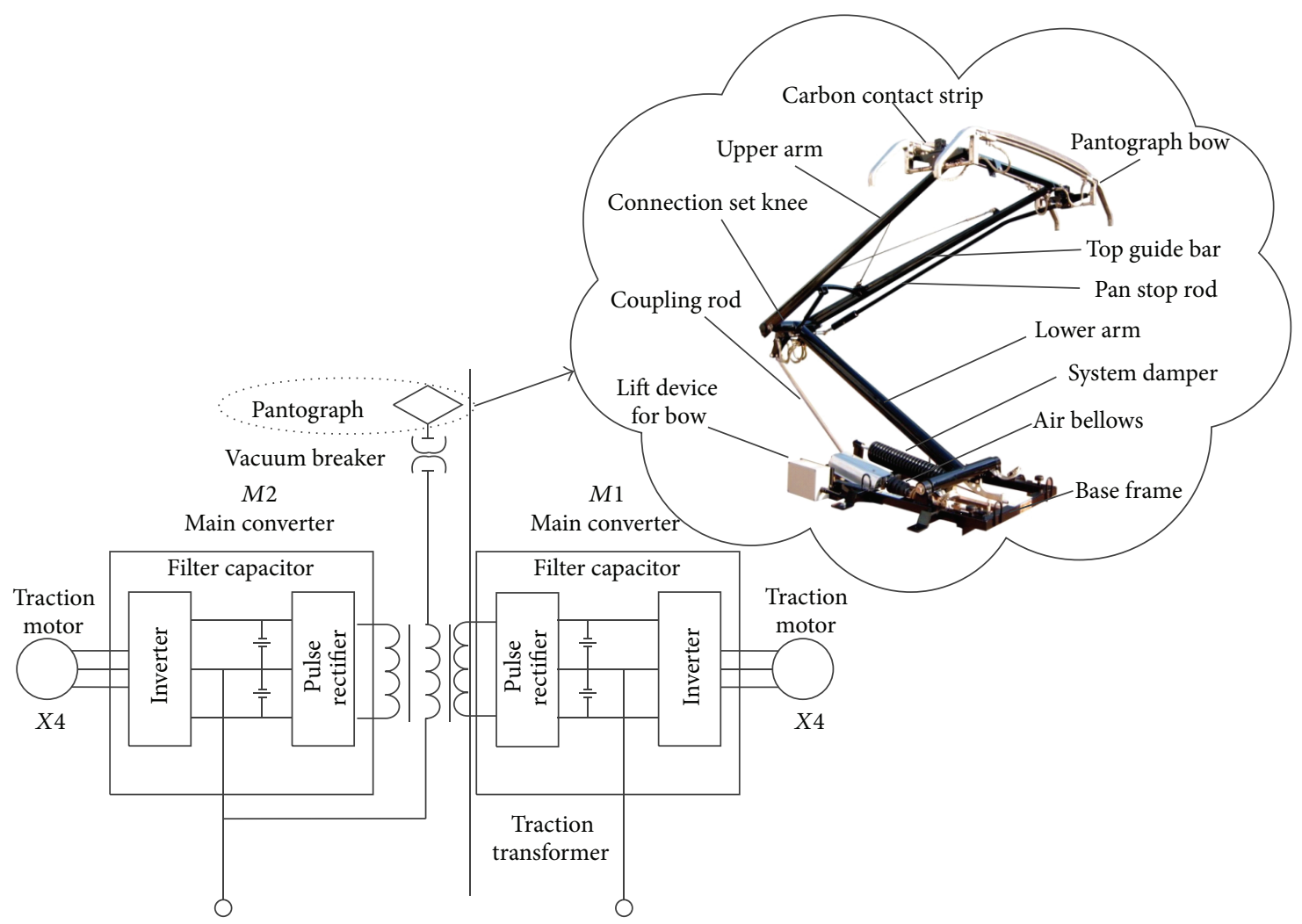

FIGURE 1: Working principle of traction drive systems and pantograph structure in a CRH EMU train.

TABLE 1: Structural parameters of the pantograph on a CRH EMU train.

\begin{tabular}{lccc}
\hline Structural parameters & Values & Structural parameters & Values \\
\hline Model & DSA250 & Vent lifting height & 3000 mm (including insulator) \\
Structure & Single arm pantograph & Maximal action height & 2800 mm (including insulator) \\
Rated voltage & $25 \mathrm{kV}$ & Minimal action height & $888 \mathrm{~mm}$ (including insulator) \\
Rated current & $1000 \mathrm{~A}$ & Folded height & $593 \mathrm{~mm}$ (including insulator) \\
Weight & $115 \mathrm{~kg}$ & Contact pressure & $70 \mathrm{~N} \pm 5 \mathrm{~N}$ \\
Ambient temperature & $-40^{\circ} \mathrm{C}+60^{\circ} \mathrm{C}$ & Design life & 30 years \\
\hline
\end{tabular}

(i) Pantograph bellows are damaged.

(j) The pantograph's carbon skateboard leaks.

(iv) Failure Mode 4. Pantograph cannot be lowered.

Probable cause:

Contacts in the pantograph's rising relay have adhered.

(v) Failure Mode 5. Pantograph cannot pass neutral section automatically.

Probable cause:

Terminal RXCB board has failed.

(vi) Failure Mode 6. Supporting insulator is damaged.

Probable causes: (a) Surface defects created during manufacture.

(b) Insulator being burned by electrical arcing.

(c) Fog flashovers discharged because of high humidity or dust.

(d) Fatigue cracks.

(e) Insulator being struck by foreign objects.

(vii) Failure Mode 7. Pantograph descends automatically.

Probable causes:

(a) Pressure air ducts in the pantograph leak.

(b) White air ducts connected with the pantograph leak.

(c) Air ducts in the pantograph control valve board leak.

(d) Pantograph's bellows are damaged. 
(e) The pantograph's carbon skateboard leaks.

(f) Incorrect manipulation.

(g) Pantograph descending loop is instantly energized by electromagnetic interference.

(h) Pressure from the catenary is too low.

(i) There has been discharge between the pantograph bracket and the roof.

(viii) Failure Mode 8 . The catenary voltage transformer is damaged.

Probable causes:

(a) Struck by foreign objects.

(b) Burned by electrical arcing.

(c) Quality control issues in components.

2.3. Fault Tree Modelling of the Pantograph. Fault tree analysis (FTA) is one type of FMEA, which is integrated with mechanics, graph theory, optimization theory, and artificial intelligence techniques [29]. It has been widely applied in many fields including aerospace systems, atomic reactors, large-scale equipment, and electronic computer systems. FTA can indicate causal relationships between complex faults and can be useful for logical analysis and diagnosis of complex system faults. It is a systematic way to assess the reliability of complex systems both qualitatively and quantitatively.

FTA is a systematic risk analysis method that deals with the occurrence of an undesired event. The fault event that analysts do not expect to happen is usually the focus of the FTA method. Analysts apply top-down logic to find all direct and indirect fault events relating to the incident. They can then establish logical relationships between the events, form a fault tree, and undertake quantitative or qualitative analysis. In the process above, the focus fault event is the top event. Selection of the top event is crucial to fault tree modelling. If the top event is too general, it is difficult to analyse the fault tree. On the other hand, if the event is too specific, the fault tree will fail to show the causal relationships of the system fully. In general, the failure of the system analyzed will be selected as the top event.

Combined with analysis of the structures and working principles of pantographs on CRH-trains, a pantograph fault tree has been established as shown in Figure 2. " $T$ " represents the top event, " $M$ " represents the intermediate event, and " $X$ " represents the basic event.

Meanings of events in Figure 2 are shown as follows.

$T$ : pantograph fails to work,

M1: pantograph rises to an abnormal position,

M2: pantograph rises normally, but the MON does not show it correctly,

M3: pantograph cannot rise,

M4: pantograph cannot be lowered,

M5: pantograph cannot pass neutral section automatically,

M6: supporting insulators are damaged,
M7: pantograph descends automatically,

M8: the catenary voltage transformer is damaged,

M9: pressure of the auxiliary air cylinder is too low,

$X 1$ : two EMUs are attached together,

$X 2$ : spacing of two pantographs is less than $190 \mathrm{~m}$,

$X 3$ : the pressure sensors do not work properly,

$X 4$ : the pressure switches have failed,

$X 5$ : EGS is closed,

$\mathrm{X} 6$ : VCB is closed,

$X 7$ : in the cab switchboards at both ends, [Pantograph.VCB] NFB is in the OFF position,

$X 8$ : in the running switchboards of the number 4 and number 6 cars (CRH-200, CRH-300)/number 4 and number 13 cars (CRH long-distance seat car, $\mathrm{CRH}$ long-distance sleeper car), [Pantograph Rising] NFB is in the OFF position,

$X 9$ : pressure air ducts of the pantograph leak,

$X 10$ : air ducts in the pantograph control valve board leak,

X11: pantograph bellows are damaged,

$X 12$ : pantograph's carbon skateboard leaks,

$X 13$ : white air ducts connected with the pantograph leak,

$X 14$ : contacts in the pantograph's rising relay have adhered,

$X 15$ : terminal RXCB board has failed,

$X 16$ : surface defects due to manufacturing errors,

$X 17$ : burned by electrical arcing,

$X 18$ : fog flashovers discharge,

$X 19$ : fatigue cracks,

$X 20$ : struck by foreign objects,

$X 21$ : driver error,

$X 22$ : pantograph descending loop is instantly energized by electromagnetic interference,

$X 23$ : discharge between the pantograph bracket and the roof,

$X 24$ : pressure of the catenary is too low,

$X 25$ : burned by electrical arcing,

$X 26$ : air ducts of the air compressor are damaged,

$X 27$ : the air compressor fails to work,

$X 28$ : MR ducts are damaged,

X29: main air cylinder leaks.

The fault tree in Figure 2 shows the causal relationships between the pantograph fault events, improving the accuracy and efficiency of fault diagnosis. However, the modelling power of FTA is limited to the static evaluation of a single criterion at a time and cannot describe dynamic system 


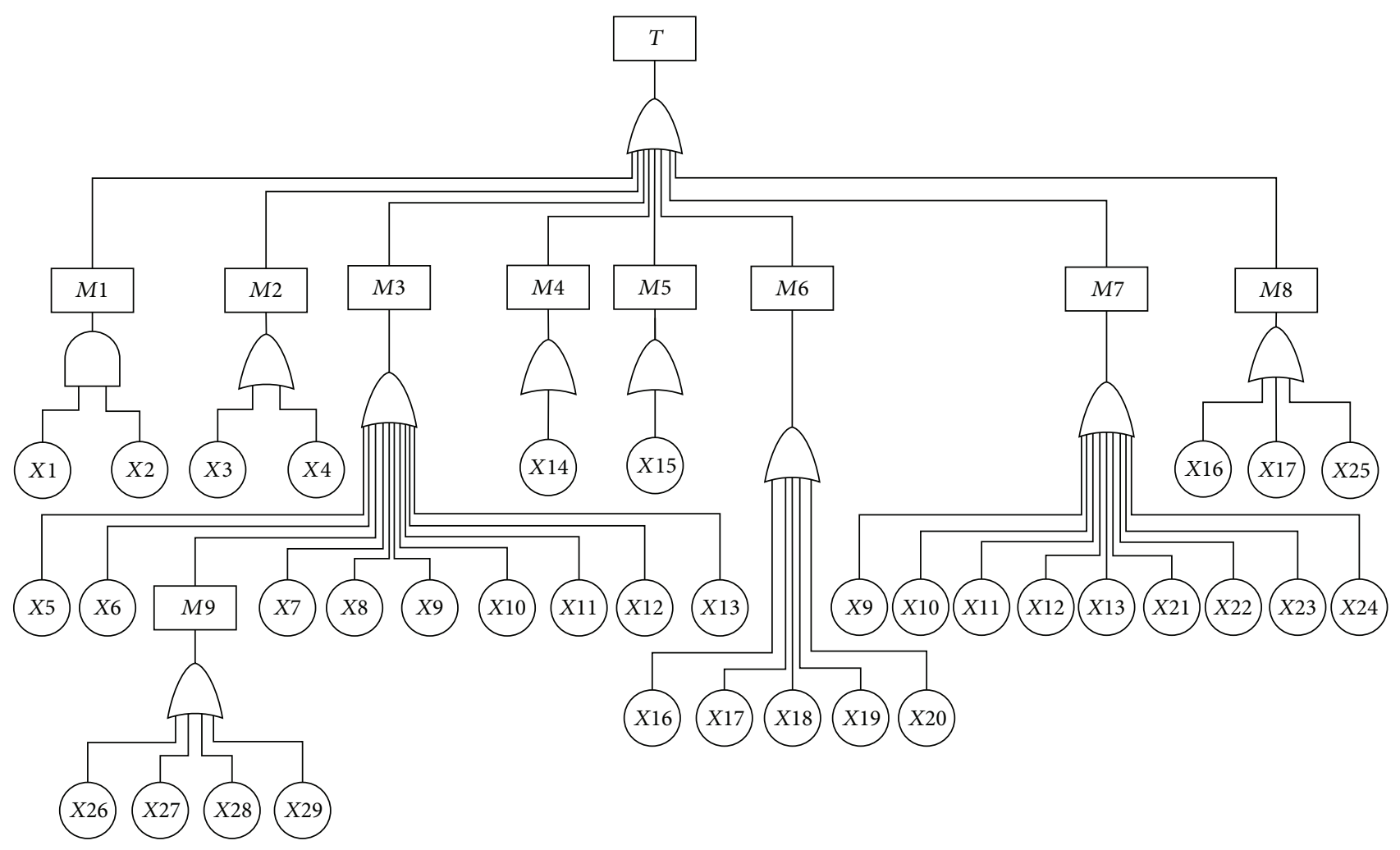

FIGURE 2: FTA modelling of the pantograph-type current collector.

behavior. It cannot show the transmission and evolution of faults in the system. It also needs too much computation to solve the minimal cut sets of the large-scale fault tree, which may lead to combinatorial explosion because of Nondeterministic Polynomial (NP) problems. For example, if $(i=$ $1,2, \ldots, n)$ is set to the $i$ th minimal cut set of the fault tree, then the top event can be expressed as

$$
T=\bigcup_{i=1}^{n} C_{i} .
$$

The occurrence probability of the top event can be expressed as

$$
P(T)=P\left(\bigcup_{i=1}^{n} C_{i}\right)
$$

The logical relationship between the basic events and the minimal cut set is and. If the probabilities of the basic events are known, the occurrence probability of the minimal cut set can be expressed as

$$
P\left(C_{i}\right)=P\left(\bigcup_{j=1}^{k} x_{i}\right)
$$

Based on the occurrence probabilities of the minimal cut sets, the occurrence probability of the top event can be expressed as

$$
\begin{aligned}
P(T)= & \sum_{i=1}^{n} P\left(C_{i}\right)-\sum_{i<j=2}^{n} P\left(C_{i} C_{j}\right)+\cdots \\
& +(-1)^{m-1} P\left(\bigcup_{i=1}^{n} C_{i}\right)
\end{aligned}
$$

According to the equation above, the process of determining the probability of the top event consists of $2^{n-1}$ parts. There are 28 minimal cut sets for the pantograph fault tree in Figure 2, so it needs $2^{27}$ parts to calculate the probability of the top event, which means $134,217,728$ items in all. The combinatorial explosion of the calculation will make quantitative analysis difficult. In addition, the FTA method is generally for static analysis and cannot reflect dynamic changes in multiple states in the system.

The dynamic and structural properties of Petri nets are used to simplify and analytically calculate the pantograph fault tree. Relational matrix analysis is used to solve the minimal cut set equation for the fault tree. Based on the established state equation of Petri nets, initial token and enabletransfer algorithms are used to express the transfer process of faults mathematically. 
TABLE 2: Petri net expression of the FTA logic gates.

Fault tree expression ${ }^{\text {AND logic gate }}$ Petri net expression

\section{Petri Nets Modelling and Relational Matrix Analysis}

3.1. Petri Nets Modelling of the Fault Tree. The Petri net was proposed in 1962 by Petri to express an information flow model for reticular structures [30]. It is a mathematical and graphical analysis tool to express the static structures and dynamic changes in a system [31-33].

In a Petri net, the system state is indicated by "O." A change in state is indicated by "I." An ordered pair is indicated by a directed arc " $\rightarrow$ " and a token is indicated by "๑." The Petri net expressions of the FTA logic gates are shown in Table 2. The transform between Petri net modelling and the fault tree is shown in Figure 3.

One Petri net can be defined in a sextuple $N=$ $\left(P, T, I, O, M, M_{0}\right)$. The six elements should meet the following conditions.

(1) $P=\left\{P_{1}, \ldots, P_{n}\right\}$ is a finite set of places. $n>0$ is the number of places.

(2) $T=\left\{t_{1}, \ldots, t_{m}\right\}$ is a finite set of transactions. $m>0$ is the number of transactions. $P \cap T=\varnothing$.

(3) $I: P \times T \rightarrow N$ is the input function, defining the set of repetitions or the weight of the directed arcs from set $P$ to set $T$. $N=\{0,1, \ldots\}$ is a set of nonnegative integers.

(4) $\mathrm{O}: T \times P \rightarrow N$ is the output function, defining the set of repetitions or the weight of the directed arcs from set $T$ to set $P . N=\{0,1, \ldots\}$ is a set of nonnegative integers.

(5) $M: P \rightarrow N$ is the set of the identification distribution of every place.

(6) $M_{0}: P \rightarrow N$ is the set of the initial identification distribution of every place.

As shown in Figures 2 and 3, the top event is replaced by the top place in the Petri net. All the probable events that may lead to the top place are represented as middle places or basic places. The logical gates of the fault tree are denoted by Transaction and Directed arc. Repeating events in the fault tree no longer exist in Petri net modelling.

Petri net modelling avoids repeating basic events and can achieve a $20 \%$ decrease in the number of places. The simplifying effect is more obvious in large-scale systems.
3.2. Relational Matrix Analysis in Solving the Minimal Cut Sets. Besides simplifying the fault tree, a Petri net also effectively overcomes the shortcomings of the traditional FTA in solving the minimal cut sets. The process of Petri net modelling can be undertaken more easily on a computer.

The structure of the Petri net can be translated into a matrix representation. The value of the input function from place $P$ to transaction $t$ is a nonnegative integer $\omega$, recorded as $I(P, t)=\omega$, represented by a directed arc from $P$ to $t$ with the side note. The value of the output function from transaction $t$ to place $P$ is a nonnegative integer $\omega$, recorded as $O(P, t)=\omega$, represented by a directed arc from $t$ to $P$ with the side note $\omega$. The side note is omitted when $\omega=1$. The directed arc is also omitted when $I(P, t)=0$ or $O(P, t)=0$. I and $O$ can both be represented as $n \times m$ nonnegative integer matrixes. The difference between $O$ and $I$ is called the relational matrix, recorded as $A=O-I$.

The steps for solving the minimal cut sets using the relational matrix method are as follows.

Step 1. Find out the row consisting only of " 1 " and " 0 " in the relational matrix A. This will be assigned the top place, with only inputs and no outputs.

Step 2. Search for "-1" by column from 1 in the top place. Any row corresponding to " -1 " will be regarded as an input to the top place. If there are multiple " -1 " values in the column, then there will be multiple inputs for the same transaction. The logical relationship between the inputs is AND.

Step 3. Search for "1" by row from "-1" determined in Step 2. Rows including " 1 " will be regarded as occupying a middle place. Then search repeatedly, following the second step, until a row without " 1 " is located, which will become a basic place. If there are multiple occurrences of " 1 " in the row, the logical relationship between the places corresponding to "1" will be OR.

Step 4. Continue to search following the second step and the third step until all the bottom basic places are located.

Step 5. Expand the bottom places according to the logical relationships $A N D$ and $O R$. Then obtain all the cut sets for the system.

Step 6. Find the minimal cut sets according to the Boolean absorption rate or the prime number method. 


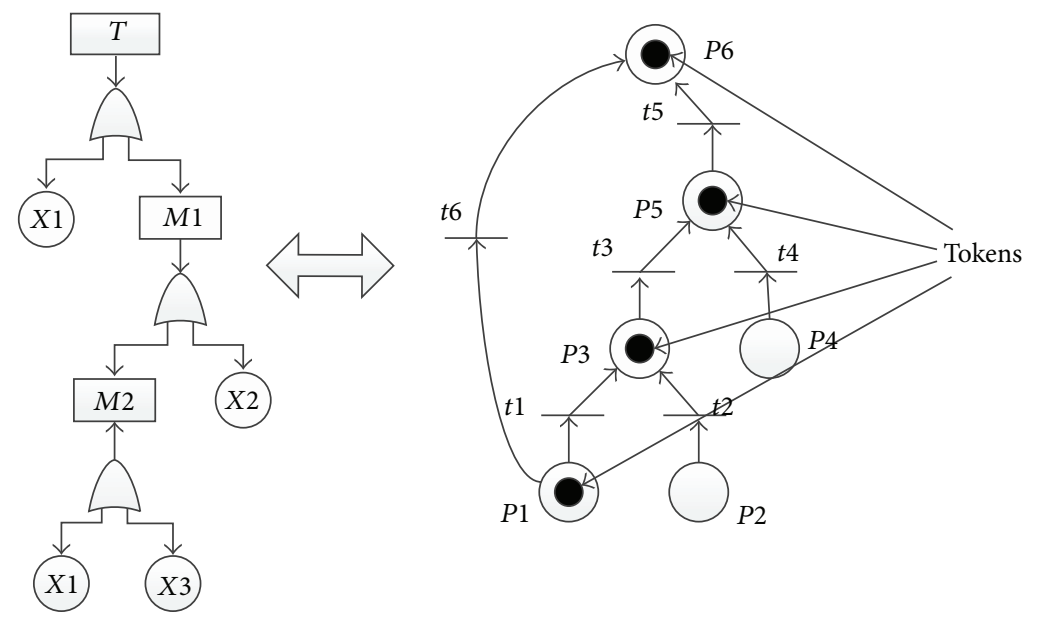

Figure 3: Transform between Petri net modelling and the fault tree.

The fault tree in Figure 3 is used as an example to illustrate solving the minimal cut sets with a relational matrix. Respectively solve the input matrix $I$, the output matrix $O$, and the relational matrix $A$ according to the steps above:

$$
\begin{aligned}
I & =\left[\begin{array}{cccccc}
1 & 0 & 0 & 0 & 0 & 1 \\
0 & 1 & 0 & 0 & 0 & 0 \\
0 & 0 & 1 & 0 & 0 & 0 \\
0 & 0 & 0 & 1 & 0 & 0 \\
0 & 0 & 0 & 0 & 1 & 0 \\
0 & 0 & 0 & 0 & 0 & 0
\end{array}\right], \\
O & =\left[\begin{array}{cccccc}
0 & 0 & 0 & 0 & 0 & 0 \\
0 & 0 & 0 & 0 & 0 & 0 \\
1 & 1 & 0 & 0 & 0 & 0 \\
0 & 0 & 0 & 0 & 0 & 0 \\
0 & 0 & 1 & 1 & 0 & 0 \\
0 & 0 & 0 & 0 & 1 & 1
\end{array}\right], \\
A & \left.=O \begin{array}{ccccccc}
-1 & 0 & 0 & 0 & 0 & -1 \\
0 & -I & -1 & 0 & 0 & 0 & 0 \\
1 & 1 & -1 & 0 & 0 & 0 \\
0 & 0 & 0 & -1 & 0 & 0 \\
0 & 0 & 1 & 1 & -1 & 0 \\
0 & 0 & 0 & 0 & 1 & 1
\end{array}\right] .
\end{aligned}
$$

3.3. Comparison between Petri Net Modelling and Fault Tree Modelling. The complexity of quantitative analysis depends on the number of nodes and the logical gates in a fault tree. Comparing Figures 3 and 4 to Figure 2, we can see that there are two repeating basic events and seven repeating events in two traditional fault trees. The repetition rates can, respectively, reach $17 \%$ and $24 \%$. These repeating events do not exist in the corresponding Petri net modelling. The building of the fault tree needs 6 types of elements which involve basic events, intermediate events, top events, AND logical gate,
OR logical gate, and a relation line, while the building of the Petri net model needs only three types of elements involving places, transactions, and directed arcs. Though the fault tree in Figure 2 seems to provide better visualization than the Petri nets in Figure 4, there are more elements and repeating events in the fault tree. The relationships among fault events in the fault tree are more complex. The reduction of building elements and repeating events make the Petri net modelling more convenient and efficient than fault tree modelling.

In addition, Petri nets are graphical and mathematical tools for modelling systems and their dynamics, while fault tree modelling cannot be updated in a timely manner when the system changes. The relationship between fault events is displayed statically in a fault tree. When the failure status of a system evolves from one subsystem/component to other subsystems/components, Petri net modelling is more intuitive and more effective than fault tree modelling because of the use of token transferring and enable-transferring processes. This process in Petri nets can be described by mathematical matrix computations, which is easier to handle by computer (as shown in Section 4).

\section{Dynamic Transition and Diagnosis of Pantograph Faults}

4.1. Place Mark and Enable-Transfer Algorithm of the Petri Net. A Petri net consists of places "O," transactions "I," directed arcs " $\rightarrow$," and tokens of places " logical descriptions of the system states, and the transactions represent the arising of system events. Relational matrixes and state equations are major tools for Petri net analysis:

$$
M_{k+1}=M_{k}+A^{T} X_{k}, \quad k=1,2, \ldots
$$

In (6), $M_{k}$ is the initial identification set of the system faults before ignition. $M_{k+1}$ is the result identification set of the system faults after ignition. $A^{T}$ is the relational matrix and $X_{K}$ is the transfer sequence for ignition. The 


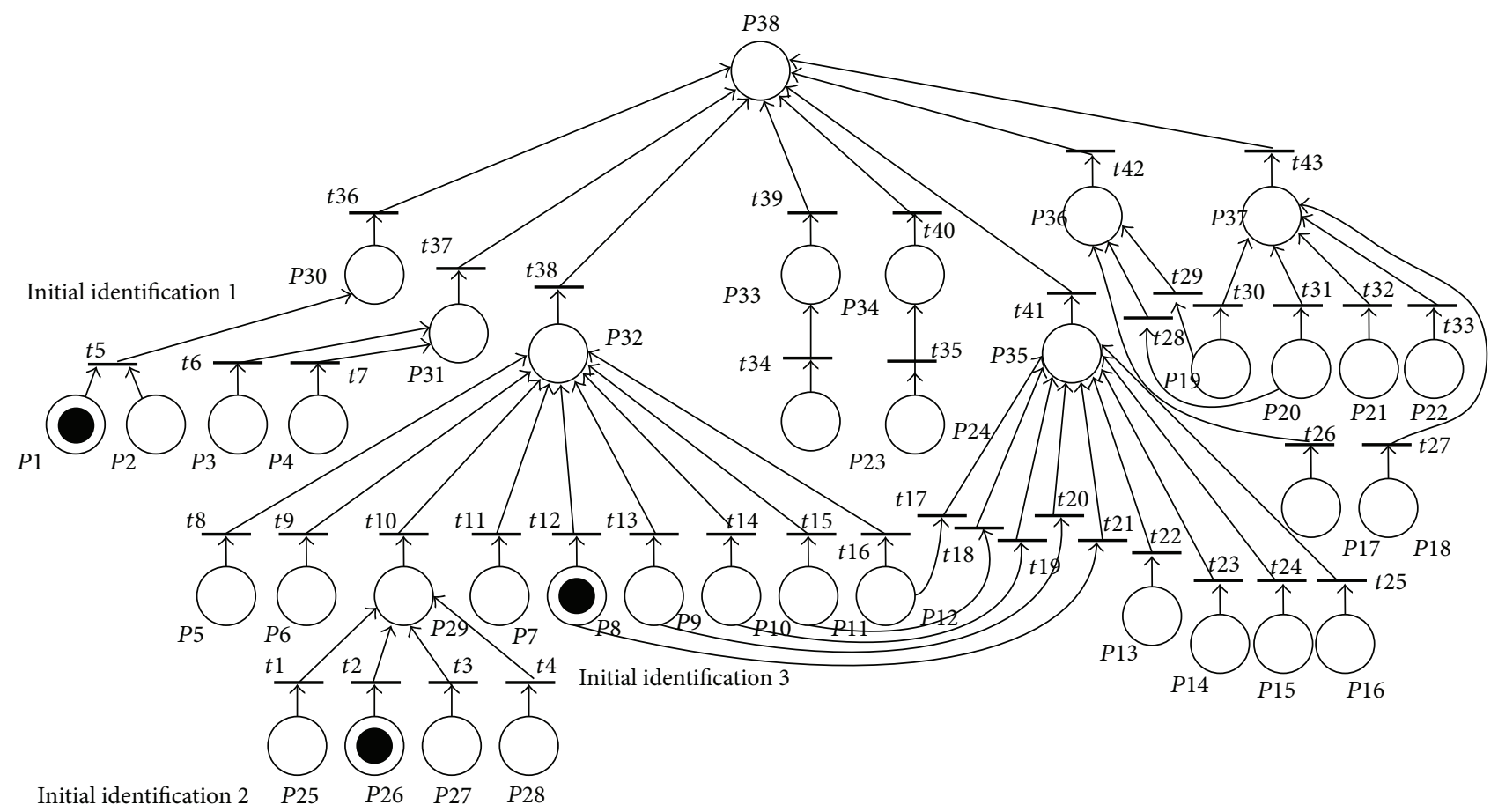

FIgURE 4: Transfer of tokens in the Petri net of the CRH EMU pantograph.

initial identification $M_{0}$ will convert to $M_{d}$ after the ignition sequence $X_{0}, \ldots, X_{d}$. The process can be expressed as

$$
M_{d}=M_{0}+A^{T} \sum_{k=0}^{d-1} X_{k}
$$

If the element representing the top place is not less than 1 in $M_{d}$ after a series of transfers, the fault event represented by the top place will occur.

\subsection{Dynamic Transition and Diagnosis of Pantograph Faults.} In the process of fault diagnosis based on the use of a Petri net, the initial identification of the input place is regarded as the initial symptom of the event occurrence. A token is assigned to the input place if the symptom appears; otherwise the place is assigned a null value. The future states and final identification of the system can be circularly solved through the state equations and transfer sequences, until the token value of the target place is found. The fault occurs when the number of tokens in the target place is not zero.

For the AND gate in the Petri net, the tokens cannot transfer downward to the next level if there are empty input places. That is to say, for an $A N D$ gate, the fault will transfer downward only when all the events of the input places occur. For the $O R$ gate in a Petri net, the tokens will transfer downward to the next level place if at least one input place contains tokens. According to the rules of the network theory, the next level place will get two tokens if the two input places both contain tokens. Regardless of the number of tokens, the occurrence of the fault that is represented by the target place is only related to whether there are tokens in the target place. Transfer of the token in the Petri net of the CRH EMU pantograph is shown in Figure 4.
As shown in Figure 4, $P 1$ represents "two EMUs attached together," $P 2$ represents "spacing of two pantographs of less than $190 \mathrm{~m}$," P8 represents "pantograph bellows that are damaged," P26 represents "MR ducts that are damaged," $P 29$ represents "pressure in the auxiliary air cylinder that is too low," P30 represents "pantograph rise to an abnormal position," P32 represents "pantograph that cannot rise," P35 represents "pantograph that descends automatically," and P38 represents "pantograph that fails to work." Three representative fault transfer paths were selected to study the transfer expression of pantograph faults in the Petri net. The initial identification sets of the three paths were, respectively, recorded as $M_{0}^{1}, M_{0}^{2}$, and $M_{0}^{3}$ :

$$
\begin{aligned}
& M_{0}^{1}=[1, \underset{2 \leq i \leq 38}{1, \ldots, 0}]^{T}, \\
& M_{0}^{2}=\left[\begin{array}{c}
0, \ldots, 0,1 \leq i \leq 25 \\
1,0, \ldots, 0 \\
27 \leq i \leq 38
\end{array}\right]^{T} \text {, } \\
& M_{0}^{3}=[\underset{1 \leq i \leq 7}{0, \ldots, 0}, \underset{9 \leq i \leq 38}{0, \ldots, 0}]^{T} .
\end{aligned}
$$

The ignition sequences of the three paths were, respectively, recorded as $X_{0}^{1}, X_{0}^{2}$, and $X_{0}^{3}$ :

$$
\begin{aligned}
& X_{0}^{1}=[\underset{1 \leq i \leq 4}{0, \ldots, 0} \underset{1,0, \ldots, 0}{1 \leq i \leq 43}]^{T}, \\
& X_{0}^{2}=[0,1, \underset{3 \leq i \leq 43}{0, \ldots, 0}]^{T} \text {, } \\
& X_{0}^{3}=[\underset{1 \leq i \leq 11}{0, \ldots, 0} \underset{12 \leq i \leq 21}{0,1}, \underset{23 \leq i \leq 43}{0}]^{0} .
\end{aligned}
$$


The input matrix $I$, output matrix $O$, and the relational matrix $A^{T}$ of the Petri net are all $38 \times 43$ matrixes which can be solved using the relational-matrix method:

$$
\begin{aligned}
& A^{T}=O-I=\left(\begin{array}{ccc}
a_{11} & \cdots & a_{1 n} \\
\vdots & \ddots & \vdots \\
a_{m 1} & \cdots & a_{m n}
\end{array}\right), \\
& a_{i j}=O_{i j}-I_{i j} \quad 1 \leq i \leq 38,1 \leq j \leq 43 .
\end{aligned}
$$

In (10), $m=38, n=43$ :

$$
\begin{aligned}
& M_{1}^{1}=0, \\
& M_{2}^{1}=0 .
\end{aligned}
$$

As (12) shows, tokens cannot transfer downward to the next level in the first path. Thus there is no token in the top place at the end of the procedure, meaning that the fault event represented by $P 38$ does not occur.

In the second path, $M_{1}^{2}, M_{2}^{2}$, and $M_{3}^{2}$ are as follows:

$$
\begin{aligned}
& M_{1}^{2}=[\underbrace{0, \ldots, 0}_{1 \leq i \leq 28}, 1, \underbrace{0, \ldots, 0}_{30 \leq i \leq 38}], \\
& M_{2}^{2}=[\underbrace{0, \ldots, 0}_{1 \leq i \leq 31}, 1, \underbrace{0, \ldots, 0}_{33 \leq i \leq 38}], \\
& M_{3}^{2}=[\underbrace{0, \ldots, 0}_{1 \leq i \leq 37}, 1] .
\end{aligned}
$$

As (13) shows, the tokens in place $P 26$ are transferred into place $P 29$ after the first ignition and then transferred into P32 after the second ignition. Finally, the tokens are transferred into the top place P38 after the third ignition, meaning that the top event occurs in this situation.

In the third situation, $M_{1}^{3}$ can be calculated through a single transfer calculation when the default value of the tokens in place $P 8$ is 1 :

$$
M_{1}^{3}=[\underbrace{0, \ldots, 0}_{1 \leq i \leq 7},-1, \underbrace{0, \ldots, 0}_{9 \leq i \leq 31}, 1,0,0,1, \underbrace{0, \ldots, 0}_{36 \leq i \leq 38}] .
$$

Thus, when there are two transfer paths for one token in the bottom basic place, there will be negative values in the next level state matrix after ignition if the default value of the tokens in the bottom basic place is still 1. There is no practical significance to this phenomenon. To avoid negative values, change the default value of the tokens in place $P 8$ from 1 to 2 to produce $M_{1}^{3^{\prime}}$ :

$$
M_{1}^{3^{\prime}}=[\underbrace{0, \ldots, 0}_{1 \leq i \leq 31}, 1,0,0,1, \underbrace{0, \ldots, 0}_{36 \leq i \leq 38}] .
$$

Then $M_{2}^{3}$ can be solved according to $M_{1}^{3^{\prime}}$ :

$$
M_{2}^{3}=[\underbrace{0, \ldots, 0}_{1 \leq i \leq 37}, 2] .
$$

According to $M_{2}^{3}$, there will be two tokens in the top place P28 and the top event will inevitably happen when tokens indicate the fault transfer. Thus the fault event in the top place will happen if there is at least one token in the top place. In the Petri net, if an input place is a bottom basic place containing tokens with a value of $n$ ( $n$ is a positive integer greater than 1 ), directed arcs out, then the default value of the tokens in the input place is $n$. The fault event presented indicated by the top place would will inevitably happen when there are tokens in the top place.

Failure Mode 1. When two EMUs are attached together and at the same time the spacing of the two pantographs is less than $190 \mathrm{~m}$, the pantograph will rise to an abnormal position. However, if only one condition of the two is satisfied, the pantograph will work normally.

Failure Mode 2. Damage to MR ducts causes low pressure in the auxiliary air cylinder, which means the pantograph cannot rise successfully.

Failure Mode 3. Damage to the pantograph bellows may cause the pantograph to descend automatically or possibly fail to rise. Both of the two conditions will lead to a fault in the pantograph.

The token transfer in the Petri net is consistent with the logic analysis of the faults. It can clearly and effectively describe the dynamic processes of the system faults transfer, achieving fast and efficient fault diagnosis.

\section{Conclusions}

In this study, the working principles and failure modes of pantographs on CRH EMU trains were analyzed systematically. Petri net modelling was used as a graphical modelling tool to simplify the logical relationships and events of the fault tree into a network with places and transactions as nodes. The events, logical gates, and logical relation lines of FT were transformed to places, transitions, and directed arcs, respectively. The complexity of modelling was reduced by $20 \%$ by avoiding repeating events. Thus, the process of solving the minimal cut sets was simplified, which effectively saved calculation time for the minimal cut set solutions for complex large-scale fault trees.

The changes in system states and the evolution of failures are described well by the dynamic properties of Petri net modelling. A mathematical model for the Petri net of the pantograph fault tree was established. The equivalence and correctness of the token-transfer description for fault diagnosis inference in a Petri net were verified. Three different fault paths were used to explain and verify the algorithm. The initial identification sets of the three paths were marked. 
The three corresponding ignition sequences were calculated using matrix transformations. Finally, the system status was assessed correctly using a mathematical method which can be handled easily in a computerised system.

This work analyzes the evolution of failure events of pantograph system. The process of other critical systems needs to be further examined. Future work will collect and analyse test data from other systems in CRH EMU trains to extend the methodology to cover the whole maintenance process for CRH EMU train systems.

\section{Conflict of Interests}

The authors declare that there is no conflict of interests regarding the publication of this paper.

\section{Acknowledgments}

This research was supported by the National Natural Science Foundation of China (no. 51475324). The authors would like to thank the reviewers for their constructive comments, which enhanced the quality of this paper.

\section{References}

[1] F. C. Jiao and B. J. Wang, The Management and Maintenance of Locomotive Rolling Stock, Beijing Jiaotong University Press, Beijing, China, 2013.

[2] L. L. Song, T. Y. Wang, X. W. Song, L. Xu, and D. Song, "Fault diagnosis of pantograph type current collector of CRH electric multiple units based on Petri net modelling and fault tree analysis," Chinese Journal of Scientific Instrument, vol. 35, no. 9, pp. 1990-1997, 2014.

[3] I. Aydin, M. Karakose, and E. Akin, "Anomaly detection using a modified kernel-based tracking in the pantograph-catenary system," Expert Systems with Applications, vol. 42, no. 2, pp. 938948, 2015

[4] E. Zio, "Reliability engineering: old problems and new challenges," Reliability Engineering and System Safety, vol. 94, no. 2, pp. 125-141, 2008.

[5] D. M. Shalev and J. Tiran, "Condition-based fault tree analysis (CBFTA): a new method for improved fault tree analysis (FTA), reliability and safety calculations," Reliability Engineering and System Safety, vol. 92, no. 9, pp. 1231-1241, 2007.

[6] Y. E. Senol, Y. V. Aydogdu, B. Sahin, and I. Kilic, "Fault tree analysis of chemical cargo contamination by using fuzzy approach," Expert Systems with Applications, vol. 42, no. 12, pp. 5232-5244, 2015.

[7] S. M. Lavasani, A. Zendegani, and M. Celik, "An extension to fuzzy fault tree analysis (FFTA) application in petrochemical process industry," Process Safety and Environmental Protection, vol. 93, pp. 75-88, 2014.

[8] J. H. Purba, "A fuzzy-based reliability approach to evaluate basic events of fault tree analysis for nuclear power plant probabilistic safety assessment," Annals of Nuclear Energy, vol. 70, pp. 21-29, 2014.

[9] N. Ramzali, M. R. M. Lavasani, and J. Ghodousi, "Safety barriers analysis of offshore drilling system by employing Fuzzy Event Tree Analysis," Safety Science, vol. 78, pp. 49-59, 2015.
[10] K.-C. Hyun, S. Min, H. Choi, J. Park, and I. Lee, "Risk analysis using fault-tree analysis (FTA) and analytic hierarchy process (AHP) applicable to shield TBM tunnels," Tunnelling and Underground Space Technology, vol. 49, pp. 121-129, 2015.

[11] S. Verlinden, G. Deconinck, and B. Coupé, "Hybrid reliability model for nuclear reactor safety system," Reliability Engineering and System Safety, vol. 101, pp. 35-47, 2012.

[12] N. G. Leveson and J. L. Stolzy, "Safety analysis using Petri nets," IEEE Transactions on Software Engineering, vol. 13, no. 3, pp. 386-397, 1987.

[13] A. Bobbio, G. Franceschinis, R. Gaeta, and L. Portinale, "Parametric fault tree for the dependability analysis of redundant systems and its high-level Petri net semantics," IEEE Transactions on Software Engineering, vol. 29, no. 3, pp. 270-287, 2003.

[14] R. Robidoux, H. Xu, L. Xing, and M. Zhou, "Automated modeling of dynamic reliability block diagrams using colored Petri nets," IEEE Transactions on Systems, Man, and Cybernetics. Part A:Systems and Humans, vol. 40, no. 2, pp. 337-351, 2010.

[15] D. Makajic-Nikolic, M. Vujosevic, and N. Nikolic, "Minimal cut sets of a coherent fault tree generation using reverse Petri nets," Optimization, vol. 62, no. 8, pp. 1069-1087, 2013.

[16] B. Wang, G. Tian, Y. Liang, and T. Qiang, "Reliability modeling and evaluation of electric vehicle motor by using fault tree and extended stochastic petri nets," Journal of Applied Mathematics, vol. 2014, Article ID 638013, 9 pages, 2014.

[17] T. P. K. Nguyen, J. Beugin, and J. Marais, "Method for evaluating an extended Fault Tree to analyse the dependability of complex systems: application to a satellite-based railway system," Reliability Engineering and System Safety, vol. 133, pp. 300-313, 2015.

[18] L. L. Song, T. Y. Wang, X. W. Song et al., "Fuzzy intelligent fault diagnosis of pantograph type current collector under the multi-source heterogeneous knowledge environment," Chinese Journal of Scientific Instrument, vol. 36, pp. 1283-1290, 2015.

[19] M. An, Y. Chen, and C. J. Baker, "A fuzzy reasoning and fuzzyanalytical hierarchy process based approach to the process of railway risk information: a railway risk management system," Information Sciences, vol. 181, no. 18, pp. 3946-3966, 2011.

[20] M. An, S. Huang, and C. J. Baker, "Railway risk assessmentthe fuzzy reasoning approach and fuzzy analytic hierarchy process approaches: a case study of shunting at Waterloo depot," Proceedings of the Institution of Mechanical Engineers F: Journal of Rail and Rapid Transit, vol. 221, no. 3, pp. 365-383, 2007.

[21] K. Noori and K. Jenab, "Fuzzy reliability-based traction control model for intelligent transportation systems," IEEE Transactions on Systems, Man, and Cybernetics: Systems, vol. 43, no. 1, pp. 229-234, 2013.

[22] R. I. Muttram, "Railway safety's safety risk model," Proceedings of the Institution of Mechanical Engineers, Part F: Journal of Rail and Rapid Transit, vol. 216, no. 2, pp. 71-79, 2002.

[23] L. Bouillaut, O. Francois, and S. Dubois, "A Bayesian network to evaluate underground rails maintenance strategies in an automation context," Proceedings of the Institution of Mechanical Engineers, Part O: Journal of Risk and Reliability, vol. 227, no. 4, pp. 411-424, 2013.

[24] Y. Xie, Y. Zhang, and F. Liang, "Crash injury severity analysis using Bayesian ordered probit models," Journal of Transportation Engineering, vol. 135, no. 1, pp. 18-25, 2009.

[25] S. Bernardi, F. Flammini, S. Marrone et al., "Enabling the usage of UML in the verification of railway systems: the DAM-rail approach," Reliability Engineering and System Safety, vol. 120, pp. 112-126, 2013. 
[26] Q. Mahboob and D. Straub, "Comparison of fault tree and Bayesian Networks for modeling safety critical components in railway systems," in Advances in Safety, Reliability and Risk Management: Proceedings of the European Safety and Reliability Conference (ESREL 2011), chapter 12, pp. 89-95, CRC Press, 2011.

[27] S. Washington and J. Oh, "Bayesian methodology incorporating expert judgment for ranking countermeasure effectiveness under uncertainty: example applied to at grade railroad crossings in Korea," Accident Analysis and Prevention, vol. 38, no. 2, pp. 234-247, 2006.

[28] P. Weber, G. Medina-Oliva, C. Simon, and B. Iung, "Overview on Bayesian networks applications for dependability, risk analysis and maintenance areas," Engineering Applications of Artificial Intelligence, vol. 25, no. 4, pp. 671-682, 2012.

[29] N. Khakzad, F. Khan, and P. Amyotte, "Safety analysis in process facilities: comparison of fault tree and Bayesian network approaches," Reliability Engineering and System Safety, vol. 96, no. 8, pp. 925-932, 2011.

[30] M. Gao, M. Zhou, X. Huang, and Z. Wu, "Fuzzy reasoning petri nets," IEEE Transactions on Systems, Man, and Cybernetics A: Systems and Humans, vol. 33, no. 3, pp. 314-324, 2003.

[31] G. J. Tsinarakis, N. C. Tsourveloudis, and K. P. Valavanis, "Modeling, analysis, synthesis, and performance evaluation of multioperational production systems with hybrid timed Petri Nets," IEEE Transactions on Automation Science and Engineering, vol. 3, no. 1, pp. 29-46, 2006.

[32] M. P. Cabasino, A. Giua, S. Lafortune, and C. Seatzu, "A new approach for diagnosability analysis of Petri nets using verifier nets," IEEE Transactions on Automatic Control, vol. 57, no. 12, pp. 3104-3117, 2012.

[33] Y.-S. Huang, Y.-S. Weng, and M.-C. Zhou, "Design of traffic safety control systems for emergency vehicle preemption using timed petri nets," IEEE Transactions on Intelligent Transportation Systems, vol. 16, no. 4, pp. 2113-2120, 2015. 


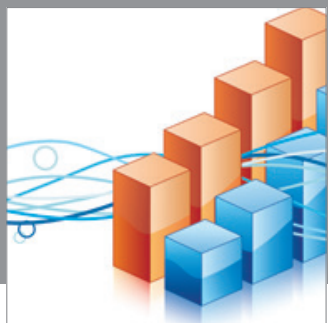

Advances in

Operations Research

mansans

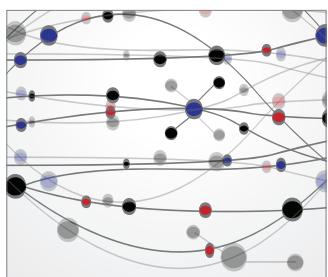

The Scientific World Journal
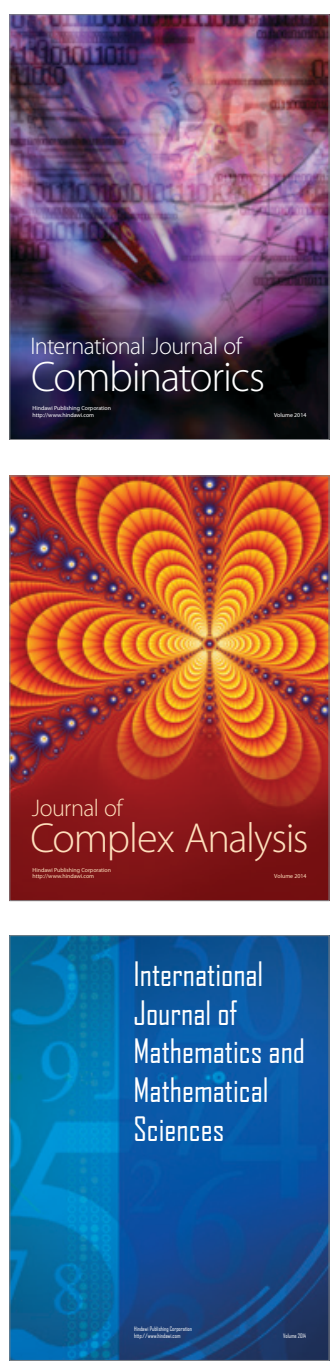
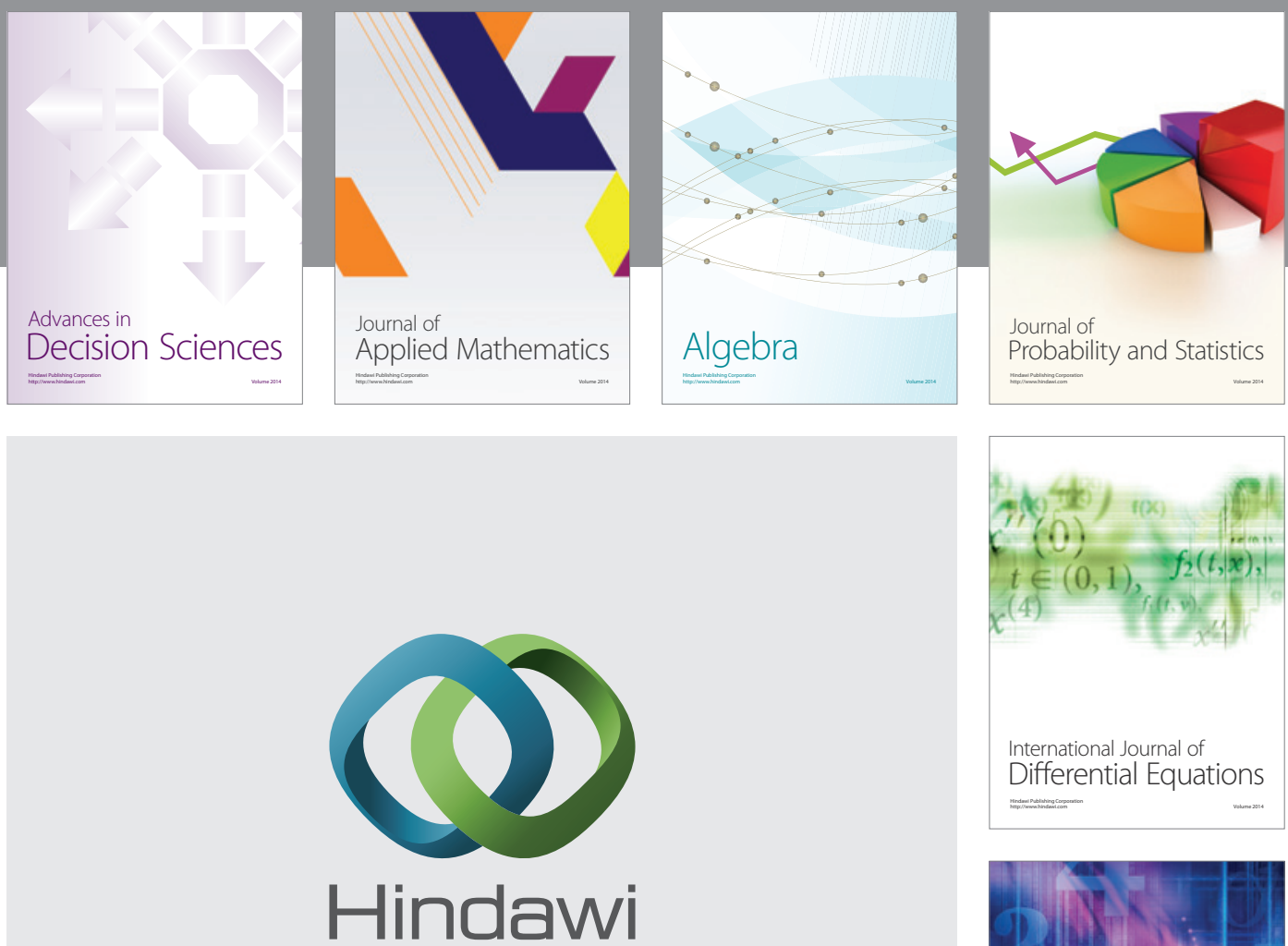

Submit your manuscripts at http://www.hindawi.com
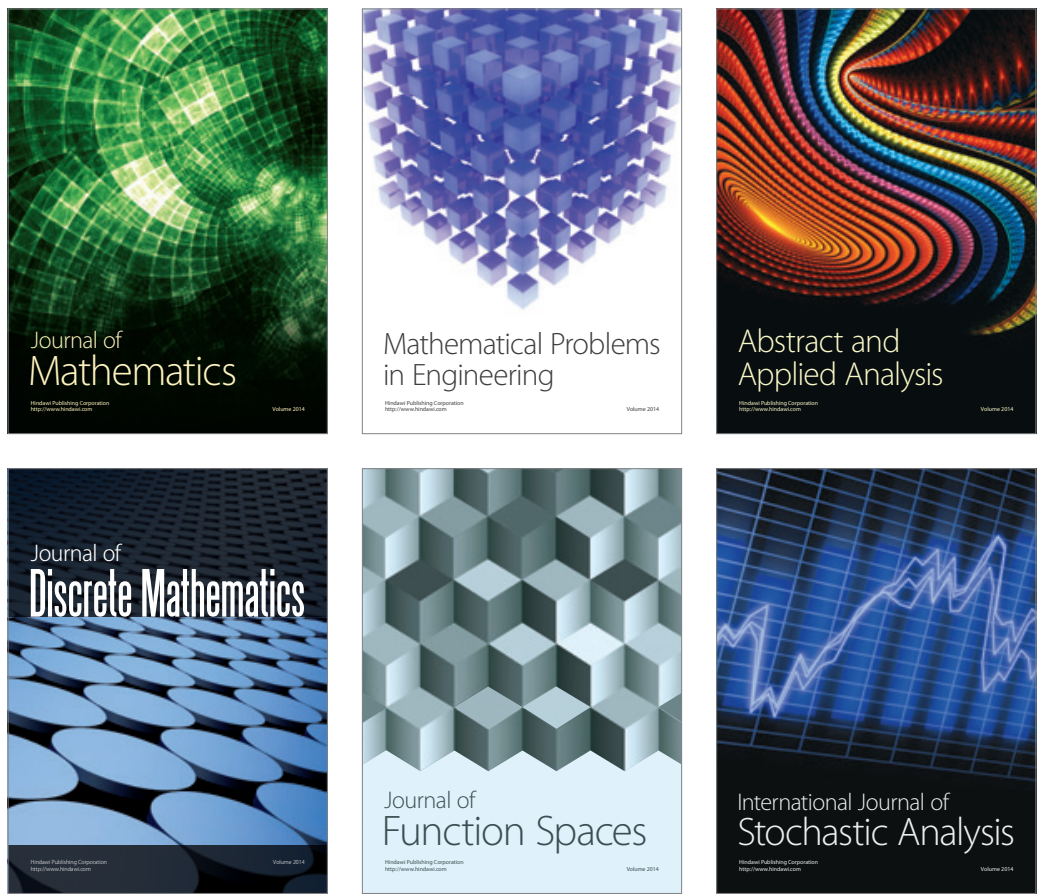

Journal of

Function Spaces

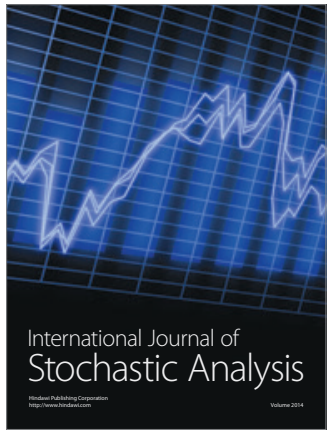

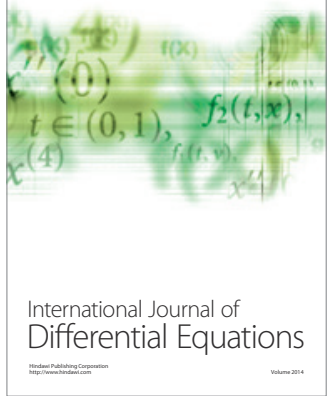
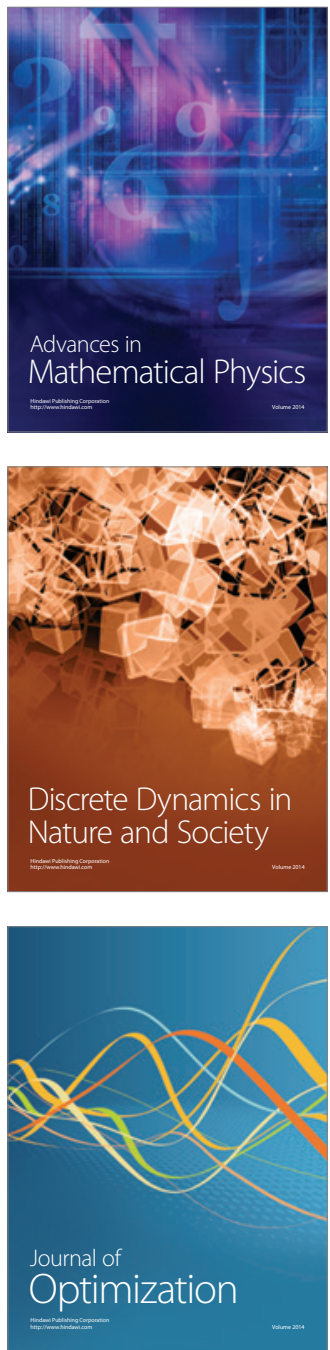